\begin{tabular}{c}
\hline \hline Juntendo \\
Research Profiles \\
\hline \hline Juntendo Medical Journal \\
2015. 61 (4), 438-439
\end{tabular}

\title{
Department of Pediatrics and Adolescent Medicine
}

\section{Principal Investigator: Toshiaki Shimizu (Professor)}

Twelve research groups exist in our department. Each subspecialty group has an active research team and carries out basic and clinical research actively.

\section{Group Leaders and Research Topics}

\section{1) Gastroenterology group}

Toshiaki Shimizu (Professor)

Takahiro Kudo (Associate Professor)

We examine the pathogenesis of mucosal immunity including inflammatory bowel disease, food proteininduced enterocolitis syndrome, and Helicobacter pylori infection, using mucosal biopsy samples. In mucosal immunologic research, cytokine profiles such as $\mathrm{TNF}-\alpha, \mathrm{IFN}-\gamma, \mathrm{IL}-17$, and IL-26, and lymphoid follicle proliferation factors have been analyzed. Furthermore, functional digestive examinations for functional gastrointestinal disorders, using gastroelectromyogram and $\mathrm{C}^{13}$-acetate breath test for gastric emptying examination, have also been performed. Reference:

1) Jimbo K, Kudo T, Shimizu T, et al: Increased expression of CXCR3 axis components and MMPs in pediatric IBD patients. Pediatr Int, 2014; 56: 873-883.

\section{2) Nutrition group}

Toshiaki Shimizu (Professor)

Tomohiro Kitamura (Assistant Professor)

The efficacy of nutritional treatment for premature and very low-birth-weight infants is examined. We also carry out clinical trials researching the safety of artificial milk supplemented with proteins and other nutrients, such as biotin and oligosaccharide. As clinical research, nutritional support team activities for inpatients are performed.

Reference:

1) Shoji H, Shimizu T, et al: Lipid profile and atherogenic indices soon after birth in Japanese preterm infants. Acta Paediatr, 2014; 103: 22-26.

\section{3) Allergy and immunology group}

Yoshikazu Ohtsuka (Associate Professor)

Mari Mori (Assistant Professor)

We analyze signaling molecules related to FceRI expression in allergic patients and investigate mechanisms of rectal bleeding in infancy in relation to the induction of tolerance. We also examine the effects of probiotics and omega-3 fatty acids in allergic and rheumatic diseases.

Reference:

1) Mori M, Ohtsuka Y, Shimizu T, et al: Outcome of infants presenting rectal bleeding: a retrospective study in a single institution. Pediatr Int, 2014; 56: 884-890.

\section{4) Cardiology group}

Masahiko Kishiro (Associate Professor)

Ken Takahashi (Associate Professor)

We examine cardiovascular function in congenital and acquired heart diseases using novel two-dimensional speckle tracking imaging and three-dimensional volume measurements. We analyze arterial function in adults with a history of Kawasaki disease. Reference:

1) Tobayama H, Takahashi K, Kishiro M, Shimizu T, et al: Analysis of arterial function in adults with a history of Kawasaki disease. J Cardiol, 2013; 61: 330-335.

\section{5) Neonatology group}

Hiromichi Shoji (Associate Professor)

Ken Hisata (Associate Professor)

We examine lipid profile, insulin sensitivity, IGF-1, and oxidative stress in preterm infants. The effects of breastfeeding on the risk factors for metabolic syndrome in preterm infants are also studied. We examine glucose metabolism soon after birth in very premature infants with low and appropriate-for-gestational-age birth weights.

Reference:

1) Suganuma H, Shoji H, Shimizu T, et al: Fat emulsion given to very low-birth weight infants increases urinary L-FABP. Pediatr Int, 2014; 56: 207-210.

\section{6) Infection group}

Ken Hisata (Associate Professor)

We examine the clinical application of nucleic acid amplification test (NAT) in pediatric infectious diseases and the molecular epidemiology of methicillin-resistant Staphylococcus aureus (MRSA) in Japan. Extendedspectrum beta-lactamases (ESBLs) for treatment, prevention, and surveillance are also studied. We further examine the metagenomics of neonatal gut microbiomes and probiotics in children.

Reference:

1) Nakao A, Hisata K, Shimizu T, et al: The clinical utility of a near patient care rapid microarray-based diagnostic test for influenza and respiratory syncytial virus infections in the pediatric setting. Diagn Microbiol Infect Dis, 2014; 78: 363-367.

\section{7) Hematology and oncology group}

Jyunya Fujimura (Associate Professor)

Sachi Sakaguchi (Assistant Professor)

We examine the molecular mechanisms of differentiation and proliferation of lymphoblasts in BCP-ALL and new tyrosine kinase-related fusion genes and their roles in $\mathrm{Ph}$-like ALL. Personalized long-term follow-up of pediatric cancer survivors using an electronic database is also performed. We further 
study the prophylaxis of febrile neutropenia by reducing chemotherapy-induced gastrointestinal mucosal injury.

Reference:

1) Tomita O, Kiyokawa N, Shimizu T, et al: Sensitivity of SNX2-ABL1 toward tyrosine kinase inhibitors distinct from that of BCR-ABL1. Leuk Res, 2014; 38: 361-370.

\section{8) Endocrinology and metabolism group}

Hidenori Haruna (Associate Professor)

Noriyuki Takubo (Associate Professor)

We examine the effect of short stature homeobox-containing (shox) gene on early embryonic growth and bone formation in zebrafish. We also examine growth disturbance in Japanese children with IBD and prolonged intracranial hypertension after recombinant growth hormone therapy.

Reference:

1) Sawada R, Shimizu T, et al: In vivo loss of function study reveals the short stature homeobox-containing (shox) gene plays indispensable roles in early embryonic growth and bone formation in zebrafish. Dev Dyn, 2015; 244: $146-156$.

\section{9) Hepatology and metabolism group}

Mitsuyoshi Suzuki (Assistant Professor)

Kei Minowa (Assistant Professor)

We examine a scoring system for the prediction of severe acute pancreatitis in children and familial and hereditary pancreatitis. The transmission route and genotype of chronic hepatitis B virus infection in children in Japan are further studied. We also examine the association of IL28B polymorphisms with virological response to peginterferon and ribavirin therapy in children and adolescents with chronic hepatitis C. Reference:

1) Suzuki M, Shimizu T, et al: Acute pancreatitis in children and adolescents. World J Gastrointest Pathophysiol, 2014; 15: 416-426.

\section{0) Neurology group}

Shinpei Abe (Assistant Professor)

Mitsuru Ikeno (Assistant Professor)

We study the utility of amplitude-integrated electroencephalography for various neurological diseases in childhood. The efficacy and safety of fosphenytoin for neonatal and infantile neurological diseases are also examined. We further study the correlation between genotype and phenotype in benign partial epilepsy in infants.

Reference:

1) Igarashi $A$, Abe $S$, Ikeno $M$, Shimizu $T$, et al: Amplitude-integrated EEG revealed nonconvulsive status epilepticus in children with non-accidental head injury. Eur J Paediatr Neurol, 2014; 18: 806-810.

\section{1) Nephrology group}

Amane Endo (Assistant Professor)

We examine the synergistic protective effects of mizoribine and angiotensin II receptor blockade on cyclosporine A nephropathy and the synergistic effect of mizoribine and a direct renin inhibitor, aliskiren, on unilateral ureteral obstruction-induced renal fibrosis in rats. Increased urinary angiotensinogen is also studied as an effective marker of chronic renal impairment in very low-birth-weight infants. Reference:

1) Endo A, Shimizu T, et al: Synergistic protective effects of mizoribine andangiotensin II receptor blockade on cyclosporine A nephropathy in rats. Pediatr Res, 2014; 75: 38-44.

\section{2) Child and adolescent mental health group}

Kyoko Tanaka (Associate Professor)

Naomi Yoshikawa (Assistant Professor)

We provide assessment, and diagnostic and treatment services for infants to adolescents with developmental and psychiatric problems. Our research interests include areas such as developmental disorders (autism spectrum disorder, ADHD, specific language impairment), high-risk infants, children with psychosomatic illness, and battered children.

Reference:

1) Tanaka K, Yoshikawa N, Shimizu T, et al: The pilot study: Sphingomyelin-fortified milk has a positive association with the neurobehavioural development of very low birth weight infants during infancy, randomized control trial. Brain Dev, 2013; 35: 45-52.

\section{3) Other references}

1) Fujitake Y, Ohtsuka Y, Shimizu T, et al: Analysis of inflammatory signals in Japanese children with Crohn's disease. Pediatr Int, 2013; 55: 753-756.

2) Shoji H, Shimizu T, et al: Oxidative stress early in infancy and neurodevelopmental outcome in very low-birthweight infants. Pediatr Int, 2014; 56: 709-713.

3) Tanaka N, Shimizu T, et al: Assessment of the developmental change in the left atrial volume using real time three-dimensional Echocardiography. Echocardiography, 2015; 32: 1131-1139.

4) Obinata K, Shimizu T, et al: Two cases of partial dominant interferon $-\gamma$ receptor 1 deficiency that presented with different clinical courses of bacille Calmette-Guérin multiple osteomyelitis. J Infect Chemother, 2013; 19: 757-760.

5) Tamaichi H, Shimizu T, et al: Ataxia telangiectasia mutated-dependent regulation of topoisomerase II alpha expression and sensitivity to topoisomerase II inhibitor. Cancer Sci, 2013; 104: 178-184.

6) Tsubahara M, Okumura A, Shimizu T, et al: Isolated growth hormone deficiency in two siblings because of paternal mosaicism for a mutation in the $\mathrm{GH} 1$ gene. Clin Endocrinol, 2012; 76: 420-424.

7) Suzuki M, Shimizu T, et al: Scoring system for the prediction of severe acute pancreatitis in children. Pediatr Int, 2015; 57: 113-118.

8) Nakazawa M, Shimizu T, Okumura A, et al: Efficacy and safety of fosphenytoin for benign convulsions with mild gastroenteritis. Brain Dev, 2015; 37: 864-867.

9) Sakuraya K, Shimizu T, et al: The synergistic effect of mizoribine and a direct renin inhibitor, aliskiren, on unilateral ureteral obstruction induced renal fibrosis in rats. J Urol, 2014; 191: 1139-1146.

10) Hosozawa M, Tanaka K, Shimizu T, et al: How children with specific language impairment view social situations: an eye tracking study. Pediatrics, 2012; 129: e1453-1460. 